\title{
REALIDADE VIRTUAL E FACILITAÇÃO NEUROMUSCULAR PROPRIOCEPTIVA COMO ABORDAGEM PARA FUNCIONALIZAÇÃO DO TEMPO DE REAÇÃO VISUAL, EQUILÍBRIO DINÂMICO E FORÇA MUSCULAR
}

\begin{abstract}
Jayne Carvalho dos Santos*, Francisco Lopes Barros*, Paulo Vinicius de Oliveira Junior*, Beatriz Christine Machado Cardoso*, Ana Carolina Gomes Martins**, Nélio Silva de Souza***, Silmar Silva Teixeira ${ }^{* * * *}$, Marco Orsini****, Victor Hugo do Vale Bastos******

Autor correspondente: Victor Hugo do Vale Bastos - victorhugobastos@ufpi.edu.br

* Discente do curso de bacharelado em Fisioterapia da Universidade Federal do Piauí (UFPI), Parnaíba, Piauí, Brasil

** Professora Assistente II do Curso de Graduação em Fisioterapia do Centro Universitário Serra dos Órgãos (UNIFESO), Mestre em Saúde Materno Infantil pela Universidade Federal Fluminense (UFF)

*** Professor Assistente II do Curso de Graduação em Fisioterapia do UNIFESO; Especialização em Geriatria e Gerontologia (UFF); Mestre em Ciências da Reabilitação (UNISUAM)

**** Professor Adjunto; Laboratório de Mapeamento Cerebral e Funcionalidade (LAMCEF/UFPI)

***** Hospital Universitário Antônio Pedro (HUAP/UFF), Niterói, Rio de Janeiro, Brasil

****** Professor Adjunto II do Departamento de Fisioterapiada UFPI, Parnaíba, Piauí, Brasil
\end{abstract}

\section{Resumo}

Introdução: A Realidade Virtual (RV) é uma interface que expõe o usuário a diversos canais sensoriais, relacionando atividades correlatas a função motora, equilíbrio, marcha e coordenação. A Facilitação Neuromuscular Proprioceptiva (FNP), por meio de decisões voluntárias, desenvolve potenciais e promove a reação de mecanismos neuromusculares como a irradiação. Objetivo: O objetivo do estudo foi avaliar a eficácia da realização de tarefas em RV associadas aos padrões de FNP em MMII de indivíduos sedentários adultos e hígidos, com análise da força muscular, tempo de reação e equilíbrio dinâmico. Materiais e métodos: A amostra contou com 18 participantes divididos aleatoriamente em três grupos ( $n=6$ ): $\mathrm{G}_{1}$ com associação de FNP e RV, G2 com FNP e $\mathrm{G}_{3}$ com RV. A experimentação se deu por cinco semanas, duas vezes por semana, utilizando-se antes e depois de cada intervenção o Make test, para avaliar a força muscular (FM), o teste de reação pedal de Nelson, para o tempo de reação visual (TRV), e o Star Excursion Balance Test, para o equilíbrio dinâmico (ED). Resultados: Ao comparar o momento posterior com o momento anterior, observou-se uma diminuição significativa no TRV para os três grupos, principalmente para $\mathrm{G}_{2}$; decréscimos significativos nos grupos $\mathrm{G}_{1}$ e $\mathrm{G}_{2}$ para a FM; no entanto, não foram encontradas alterações estatisticamente significantes para o ED em nenhum grupo. Conclusão: Os três grupos, principalmente em $\mathrm{G}_{2}$, apresentaram eficácia para 
redução do TRV, no entanto, não se mostraram eficazes para ganho significativo do ED e FM em adultos sedentários e hígidos.

Palavras-chave: Facilitação neuromuscular proprioceptiva; Tempo de reação visual; Força; Realidade virtual, equilíbrio.

\title{
VIRTUAL REALITY AND PROPRIOCEPTIVE NEUROMUSCULAR FACILITATION AS APPROACH FOR FUNCIONALIZATION OF VISUAL REACTION TIME, DYNAMIC BALANCE AND MUSCLE STRENGHT
}

\begin{abstract}
Introduction: Virtual Reality (VR) is an interface that exposes the user to different sensory channels, involving activities such as motor function, balance, gait and coordination. The Proprioceptive Neuromuscular Facilitation (PNF), through voluntary decisions, develops potential and promotes the reaction of neuromuscular mechanisms for irradiation. Objective: The aim of the study was to evaluate the effectiveness of performing tasks in VR associated with patterns of PNF in the lower limbs of sedentary adults and healthy subjects, with analysis of muscle strength, reaction time and dynamic balance. Materials and methods: The sample included 18 participants randomly assigned to three groups $(n=6): G 1$ with FNP and RV association, $G_{2}$ with PNF and $\mathrm{G}_{3}$ with VR. The trial took place for five weeks, twice a week, employing before and after each intervention the make test to assess the muscle strength (MS), the pedal reaction test Nelson, for visual reaction time (VRT), and the star excursion balance Test, for dynamic balance (DB). Results: When comparing subsequent time to the previous time there was a significant decrease in TRV for the three groups, particularly to $\mathrm{G}_{2}$; Significant decreases in the groups $\mathrm{G}_{1}$ and $\mathrm{G}_{2}$ for $\mathrm{FM}$; however, there were no statistically significant changes to the ED in either group. Conclusion: The three groups, mainly in $\mathrm{G}_{2}$, showed efficacy in reducing TRV, however, it was not effective for significant gain of ED and FM in sedentary and healthy adults.

Keywords: Virtual Reality Exposure Terapy; Neuromuscular Junction; Reaction Time; Postural Balance and Muscle Strength.
\end{abstract}

\section{INTRODUÇÃO}

A Realidade Virtual (RV) é uma interface que permite ao usuário interagir por meio de múltiplos canais sensoriais, em tempo real, com ambientes e/ ou atividades simuladas por um computador. (1) Tais $^{2}$ simulações podem ser classificadas de acordo com o nível de imersão proporcionada ao usuário em não-imersivas, semi-imersivas e imersivas. ${ }^{(2)} \mathrm{Di}$ versos são os dispositivos especiais responsáveis por fornecer essa possibilidade de interação com a RV, como óculos estereoscópicos e luvas rastrea- 
doras. ${ }^{(3)}$ Desde seu surgimento, a partir da década de 1960 , (4) a RV vem sendo utilizada em múltiplas áreas do conhecimento, havendo destaque para o ensino-aprendizagem, devido à possibilidade de simular, por meio da $\mathrm{RV}$, situações reais com riscos controlados ou sem risco algum. ${ }^{(5)} \mathrm{Na}$ área da saúde, uma das classes mais exploradas por meio da utilização da RV são as terapias, como o tratamento de distúrbios psicológicos, sequelas cognitivas e motoras, dentre outras condições. ${ }^{(6)}$ Importante salientar que a maioria dos jogos foram idealizados para entretenimento, e que são utilizados como tal e não com objetivos terapêuticos. Ressalta-se o seu baixo custo, bem como fatores motivacionais que podem facilitar a terapia quando associada a realidade virtual.

Atualmente, um dos instrumentos de RV mais utilizados na reabilitação funcional são os videogames, devido ao seu fácil acesso e ao advento dos denominados exergames, também conhecidos como jogos cinéticos, nos quais a atividade corporal vigorosa é capturada como um sinal de entrada para interação com o conteúdo digital. Eles fornecem aos indivíduos a possibilidade de desenvolver, em ambiente virtual, atividades em que os mesmos teriam mais dificuldade, se fossem realizadas em ambiente real, proporcionando a melhora da função motora, equilíbrio, marcha e coordenação. (7)

Para a fisioterapia, que tem como objeto de estudo o movimento humano, existem diversos conceitos e técnicas que podem ser empregados na reabilitação funcional, dentre estes a Facilitação Neuromuscular Proprioceptiva (FNP). A técnica utiliza contrações musculares concêntricas, excêntricas e estáticas, combinadas com resistência graduada e procedimentos facilitadores adequados, todas ajustadas para atingir as necessidades de cada paciente. ${ }^{(8)}$ A FNP parte do princípio que o indivíduo é dotado de potenciais que podem ser desenvolvidos por influência do meio ou de decisões voluntárias, promovendo a reação dos mecanismos neuromusculares por estimulação dos proprioceptores. ${ }^{(9)}$
Durante o movimento, é necessário um controle do centro de gravidade corporal enquanto se desloca sobre a base de apoio e de níveis adequados de força nos membros inferiores (MMII) para manutenção de equilíbrio estático e dinâmico. $O$ equilíbrio dinâmico (ED) é a utilização de informações externas e internas com associação da ativação muscular em resposta a perturbações da estabilidade e da capacidade de manter o corpo sobre uma base de apoio. ${ }^{(10,11)}$ Visto que o sedentarismo gera diminuição generalizada de funcionalidade corporal, ${ }^{(12,13)}$ o presente estudo teve como objetivo geral avaliar a eficácia da realização de tarefas em RV associadas às aplicações de padrões de FNP em MMII de indivíduos sedentários adultos e hígidos, com análise da força muscular (FM), tempo de reação visual (TRV) e ED. O estudo tenta suprir a lacuna que existe na relação realidade virtual enquanto tratamento fisioterapêutico e sua associação com o FNP a fim de quantificar as alterações observadas após o emprego da proposta terapêutica. Por este motivo, o estudo torna-se relevante para a comunidade cientifica.

\section{MATERIAIS E MÉTODOS}

O presente estudo enquadra-se como ensaio pré-clínico randomizado, do qual participaram indivíduos com idades entre 18 e 30 anos, saudáveis, que declararam ao menos seis meses prévios de sedentarismo e que tenham assinado o termo de consentimento livre e esclarecido (TCLE). Foram excluídos do estudo participantes portadores de distúrbios ortopédicos, cardiorrespiratórios, neurológicos, visuais e/ou vestibulares. Os procedimentos experimentais aconteceram no Laboratório de Mapeamento Cerebral de Funcionalidade (LAMCEF) da Universidade Federal do Piauí (UFPI), Campus Ministro Reis Velloso, da cidade de Parnaíba-PI, sendo realizados dois atendimentos semanais de uma hora, durante cinco semanas, totalizando dez atendimentos ao todo para cada participante. Os 15 minutos iniciais e finais de atendimento foram des- 
tinados à avaliação, enquanto o tempo restante foi destinado à intervenção.

Os participantes do estudo foram divididos aleatoriamente por conveniência em três grupos: $G_{1}$, $\mathrm{G}_{2}$ e $\mathrm{G}_{3}$; sendo o tempo de intervenção do grupo $\mathrm{G} 1$ subdivido em 15 minutos para intervenção convencional com FNP para MMII, com execução de diagonais em padrão flexor e extensor e suas derivações, em decúbito dorsal sobre uma maca, e mais 15 minutos para execução de tarefas de um jogo de RV com movimentação livre em bipedestação. Os outros dois grupos passaram os 30 minutos de intervenção sendo submetidos apenas a um tipo de procedimento, sendo $\circ \mathrm{G}_{2}$ apenas a intervenção com FNP e o $\mathrm{G}_{3}$ apenas as tarefas com RV. Todos os procedimentos realizados no estudo tiveram acompanhamento integral e auxílio do terapeuta/avaliador. Vale ressaltar que o mesmo pesquisador que avaliava era quem realizava as manobras.

Os dispositivos utilizados no estudo para proporcionar aos participantes dos grupos $\mathrm{G}_{1}$ e $\mathrm{G}_{3}$ as tarefas executadas com o uso de RV foram: um

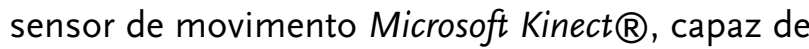
interpretar o posicionamento e a movimentação corporal do usuário no espaço, ${ }^{(14)}$ conectado a um console de videogame Xbox $360 \AA$, com imagens projetadas por um dispositivo da marca Epson, modelo PowerLite Home Cinema 8350. O jogo utilizado foi o Kinect Adventures na modalidade cume dos reflexos, cujo ambiente virtual proporciona ao jogador a possibilidade de evitar obstáculos através de movimentos corporais como saltos, agachamentos e esquivas laterais. Este jogo foi selecionado por gerar adequadamente o estímulo virtual que os pesquisadores desejavam, com a associação de movimentos que pode ser realizada em diagonal ou não como muitas das manobras de FNP principalmente nos membros superior e inferior. $\mathrm{O} \mathrm{Mi}$ crosoft Kinect $₫$ é baseado em uma câmera-sensor utilizada para controlar diretamente um jogo por meio do movimento corporal, sem a necessidade de plataformas de equilíbrio ou outros recursos. ${ }^{(15)}$ O Kinect apresenta um sensor de profundidade que permite a mensuração de padrões tridimensionais de movimento, o que viabiliza o feedback em tempo real. ${ }^{(16)}$

Para a avaliação da FM dos extensores de joeIho foi utilizado o teste de execução (Make Test), no qual os participantes permaneciam em sedestação com um esfigmomanômetro insuflado a 60 $\mathrm{mmHg}$, posicionado entre o tornozelo e um objeto estacionário e com os joelhos fletidos a $90^{\circ}$. Por meio de um estímulo verbal, tentaram realizar a extensão dessa articulação, gerando esforço máximo em contração isométrica, por três tentativas em cada membro inferior, com intervalos de 60 segundos entre uma mensuração e outra. ${ }^{(17)}$

Para avaliação do TRV dos membros inferiores foi utilizado o teste de velocidade de reação pedal de Nelson, no qual o participante, sentado em uma cadeira sobreposta a uma mesa posicionada a $2,5 \mathrm{~cm}$ de distância da parede, descalço, com o pé posicionado a uma distância da parede igual à da mesa, enquanto o examinador segura uma régua na extremidade mais afastada, colocando-a contra a parede e alinhando com o hálux do participante. A partir daí, com o participante olhando diretamente para a zona de concentração situada na extremidade superior da régua, o examinador deixa a régua cair e o participante reage tentando pressionar a mesma com a polpa do pé contra a parede. São realizadas cinco tentativas para cada participante e obtida a média da distância percorrida, posteriormente convertida em tempo (milissegundos), utilizandose a tabela de conversão tempo de reação-distância. Ao final os participantes ainda respondem a um questionário que visa avaliar se a terapêutica foi motivacional e qual o grau de dificuldade de compreensão e execução das tarefas. ${ }^{(18,19)}$

A avaliação do ED foi realizada através do Star Excursion Balance Test (SEBT) modificado, no qual é mensurada a habilidade de um indivíduo de manter-se em equilíbrio corporal com apoio unipodal enquanto realizam-se tentativas de alcance da maior distância possível com o membro contralateral em direções anterior, póstero-lateral e póstero-medial, indicadas por três linhas de $120 \mathrm{~cm}$ fixadas no chão a partir de um ponto central comum (figura 1). 


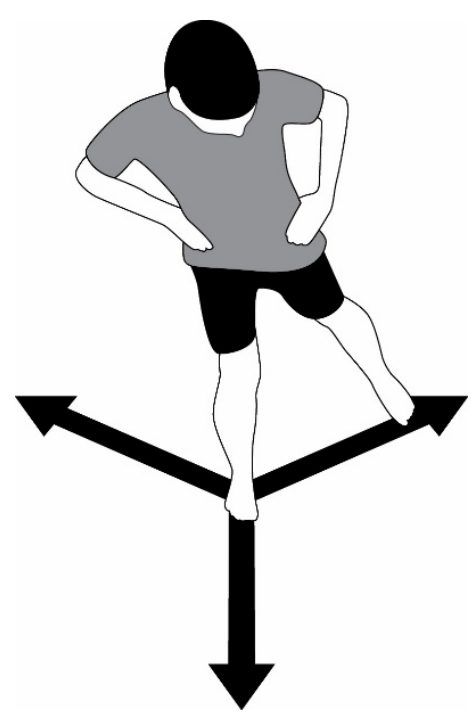

Figura 1 - SEBT modificado. Figura criada pelos autores.

Os sujeitos avaliados foram posicionados na interseção das três direções do teste em apoio unipodal e com a ponta pé do membro contralateral efetuaram toques suaves o mais distante possível em cada uma das direções. Em cada direção executou três tentativas intercalando-se o apoio alternado entre os MMII com um intervalo de 10 segundos entre cada uma das tentativas. ${ }^{(20,21)}$ Para eliminar a influência do tamanho do membro, a maior distância adquirida em cada direção foi dividida pelo tamanho do membro do indivíduo e multiplicado por 100. Em seguida realizou-se a média das porcentagens das três direções a fim de se adquirir o escore composto, o qual demonstra o desempenho geral no teste. ${ }^{(22)}$ Todos os testes foram previamente esclarecidos aos participantes. O estudo obteve aprovação do Comitê de Ética em Pesquisa da Universidade Federal do Piauí - UFPI, de acordo com o parecer 826.712 e está de acordo com os princípios éticos estabelecidos na Resolução 466/12 do CNS.

\section{RESULTADOS}

Dos 24 indivíduos convidados a participar do estudo seis foram excluídos: um por apresentar indícios de vestibulopatia durante a anamnese inicial; um por instabilidade articular de quadril durante intervenção e quatro por desistência voluntária. Assim, apenas 18 foram introduzidos no estudo. A média de idade foi de 20 anos $( \pm D P 2,1)$. A fim de compreender os efeitos do momento anterior e posterior à intervenção, de membros inferiores direito ou esquerdo utilizados e a capacidade de gerar FM dos grupos, foram realizadas análises utilizando three-way Mixed fatorial ANOVA. Os dados estavam normalmente distribuídos conforme observado no teste de Shapiro-Wilk $(p>0,05)$.

\section{TEMPO DE REAÇÃO VISUAL}

Para o TRV, pode-se observar que não houve homogeneidade na variância para MIE antes $[\mathrm{F}(2,177)=$ 6,792, $p=0,001$ ] por meio do teste de Levene para igualdade de variâncias ( $p>0,05),[F(2,177)=18,625$, $p=0,00, \eta^{2}$ parcial $\left.=0,174\right]$. Não foi encontrada interação estatisticamente significativa nas três vias entre momento, condições e grupos, mas houve a significância estatística de uma interação da via simples momento $\left[F(1)=12,528, p=0,001, \eta^{2}\right.$ parcial $=0,066]$, aceito em um nível alfa-Bonferroni ajustado de 0,025. Para os grupos, os dados do TRV no momento antes, em média seguido do desvio padrão, foram de 197,194 \pm 2,114 ms, e depois, 192,203 $\pm 1,808$ ms. No teste Post Hoc para grupo, houve uma diferença média estatisticamente significante 
entre os grupos $\mathrm{G}_{1}$ e $\mathrm{G}_{2}$ de 13,99 ms (IC 95\% 3,125 a 24,867), $\mathrm{p}=0,007$, e também ente $\mathrm{G}_{3}$ e $\mathrm{G}_{1}$ de 13,45 ms (IC 95\%, 2,581 a 24,323), $\mathrm{p}=0$ 0,010, além de um efeito principal entre $\mathrm{G}_{3}$ e $\mathrm{G}_{2}$ de $27,44 \mathrm{~ms}$ (IC 95\%, $16,578$ a 38,319$), p=0,00$.

O gráfico 1 corresponde às médias e desvio padrão do TRV de $G_{1}, G_{2}$ e $G_{3}$ nos membros direito e esquerdo, antes e depois do atendimento. No membro direito, a média encontrada antes do atendimento para $G_{1}$ foi de 200,85 ms ( \pm DP 20,13), G 2 de $184,90 \mathrm{~ms}( \pm \mathrm{DP} 21,97)$ e $\mathrm{G}_{3}$ de $207,00 \mathrm{~ms}( \pm \mathrm{DP}$ 24,46). A média encontrada depois para $G 1$ foi de $195,16 \mathrm{~ms}$ ( \pm DP 19,26), G2 de 182,00 ms ( \pm DP $23,1)$ e $\mathrm{G}_{3}$ de $213,20 \mathrm{~ms}( \pm \mathrm{DP} 25,44)$. No membro esquerdo, a média encontrada antes para $G 1$ foi de $192,72 \mathrm{~ms}( \pm \mathrm{DP} 18,79), \mathrm{G}_{2}$ foi de $180,90 \mathrm{~ms}( \pm \mathrm{DP}$ $11,95)$ e $\mathrm{G}_{3}$ de 205,60 ms ( \pm DP 31,17). Já a média no momento posterior ao atendimento para $\mathrm{G}_{1}$ foi de $190,78 \mathrm{~ms}( \pm \mathrm{DP} 13,10), \mathrm{G}_{2}$ foi de $175,60 \mathrm{~ms}( \pm \mathrm{DP}$ $20,32)$ e $\mathrm{G}_{3}$ de $207,60 \mathrm{~ms}( \pm \mathrm{DP} 22,57$ ).

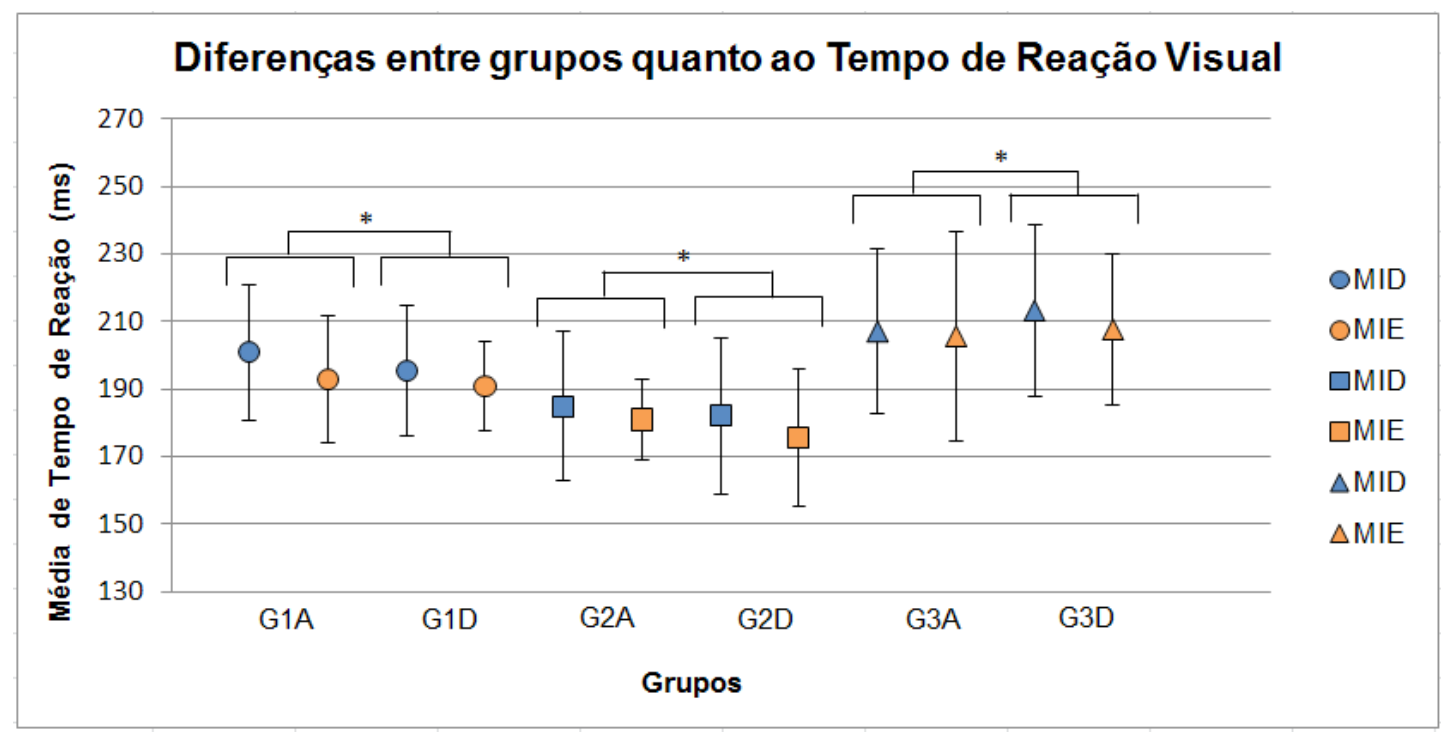

Gráfico 1 - Diferenças entre grupos quanto ao Tempo de Reação Visual

O gráfico 1 Apresenta a média e erro padrão do tempo de reação visual avaliado no momento antes e depois da intervenção para o membro inferior direito (MID), em azul, e membro inferior esquerdo (MIE), em laranja. Os grupos $\mathrm{G}_{1} \mathrm{~A}, \mathrm{G}_{2} \mathrm{~A}$ e $\mathrm{G}_{3} \mathrm{~A}$ correspondem aos grupos de associação entre FNP e RV, somente FNP e somente RV no momento anterior; enquanto $G_{1} D, G_{2} D$ e $G_{3} D$, aos mesmos grupos no momento posterior ao atendimento. $\mathrm{O}$ asterisco $(*)$ corresponde à diferença significativa com $\mathrm{p}<0,05$.

\section{EQUILÍBRIO DINÂMICO}

Após avaliação realizada pelo teste de Levene para igualdade de variâncias $(p>0,05)[F(2,177)=4,807$, $p=0,009, \eta^{2}$ parcial $\left.=0,052\right]$, pode-se observar que não houve homogeneidade na variância para MID depois $[F(2,177)=3,685, p=0,027]$ e MIE antes $[F(2,177)=4,222, p=0,016]$. Ao analisar o equilíbrio, não foi observada uma interação estatisticamente significativa nas três vias entre momento, condições e grupos, mas houve a significância estatística de uma interação nas condições e grupos $\left[F(2,177)=4,93, p=0,008, \eta^{2}\right.$ parcial $\left.=0,053\right]$. A significância estatística de um efeito principal simples para momento foi aceito em um nível alfa-Bonferroni ajustado de 0,025 . Um resultado estatisticamente significativo foi encontrado para condições de MID antes $\left[F(2,177)=4,885, p=0,009, \eta^{2}\right.$ parcial $=0,052]$, para MID depois $[F(2,177)=5,877, p=$ $0,003, \eta^{2}$ parcial $\left.=0,062\right]$, e MIE depois $[F(2,177)=$ 
6,016, $p=0,003, \eta^{2}$ parcial $\left.=0,026\right]$, mas não para o MIE antes $\left[F(2,177)=2,364, p=0,097, \eta^{2}\right.$ parcial $=0,064]$. No teste Post Hoc, houve uma diferença média estatisticamente significante entre os grupos $\mathrm{G}_{2}$ e $\mathrm{G}_{3}$ de 3,85 cm (IC 95\% o,850 a 6,866), $\mathrm{p}=0,007$.

O gráfico 2 apresenta as médias e o erro padrão da condição $G_{1}, G_{2}$ e $G_{3}$ nos membros direito e esquerdo, no momento anterior e posterior ao atendimento. Para o membro direito, a média encon- trada antes para $\mathrm{G}_{1}$ foi de $88,40 \mathrm{~cm}\left( \pm \mathrm{DP}_{5}, 04\right), \mathrm{G}_{2}$ de $90,00 \mathrm{~cm}( \pm \mathrm{DP} 7,06)$ e $\mathrm{G}_{3}$ de $86,10 \mathrm{~cm}( \pm \mathrm{DP}$ $5,16)$, enquanto a média posterior para $G_{1}$ foi de $87,84 \mathrm{~cm}( \pm \mathrm{DP} 4,36), \mathrm{G} 2$ de $90,80 \mathrm{~cm}( \pm \mathrm{DP} 6,62) \mathrm{e}$ $\mathrm{G}_{3}$ de $86,40 \mathrm{~cm}( \pm \mathrm{DP} 5,77)$. No membro esquerdo, a média encontrada antes para $G_{1}$ foi de $87,89 \mathrm{~cm}$ ( \pm DP 5,55$), \mathrm{G}_{2}$ foi de $88,40 \mathrm{~cm}( \pm \mathrm{DP} 8,29)$ e $\mathrm{G}_{3}$ de $85,60 \mathrm{~cm}( \pm \mathrm{DP} 2,83)$. Já média posterior ao atendimento na condição $G_{1}$ foi de $87,46 \mathrm{~cm}\left( \pm D^{D P} 4,8\right)$, $\mathrm{G}_{2}$ foi de $90,20 \mathrm{~cm}( \pm \mathrm{DP} 7,23)$ e $\mathrm{G}_{3}$ de $85,90 \mathrm{~cm}$ $( \pm$ DP 4,9$)$.

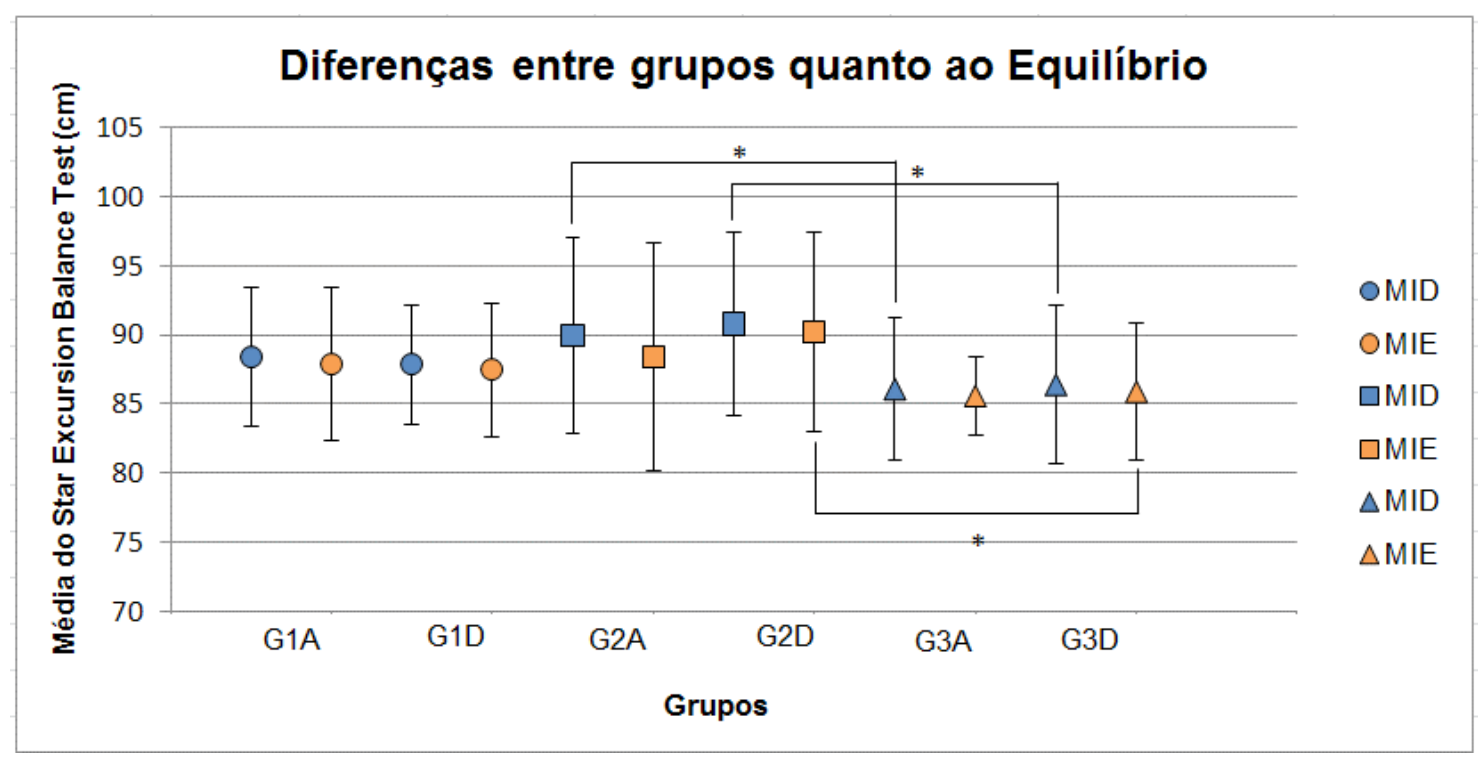

Gráfico 2 - Diferenças entre grupos quanto ao Equilíbrio

O gráfico 2 apresenta a média e erro padrão do equilíbrio avaliado no momento antes e depois da intervenção para o membro inferior direito (MID), em azul, e membro inferior esquerdo (MIE), em laranja. Os grupos $\mathrm{G}_{1} \mathrm{~A}, \mathrm{G}_{2} \mathrm{~A}$ e $\mathrm{G}_{3} \mathrm{~A}$ correspondem aos grupos de associação entre FNP e RV, somente FNP e somente RV no momento anterior; enquanto $G_{1} D, G_{2} D$ e $G_{3} D$, aos mesmos grupos no momento posterior ao atendimento. $\mathrm{O}$ asterisco (*) corresponde à diferença significativa com $p<0,05$.

\section{FORÇA MUSCULAR}

Para FM, foi observado homogeneidade das variâncias $\left[F(2,177)=1,961, p=0,144, \eta^{2}\right.$ parcial $\left.=0,022\right]$, avaliado pelo teste de Levene para igualdade de variâncias $(p>0,05)$. Após a análise, observou-se uma interação estatisticamente significativa nas três vias entre momento, condições e grupos. $[F(2,177)$ $=5,605, p=0,004, \eta^{2}$ parcial $\left.=0,060\right]$, e a significância estatística de um efeito principal simples foi aceito em um nível alfa-Bonferroni ajustado de 0,025 . Houve uma interação estatisticamente significativa simples de mão dupla entre o momento e as condições para grupo $\mathrm{G}_{2}[\mathrm{~F}(1,59)=9,376, \mathrm{p}=$ $0,003]$, mas não para grupo $\mathrm{G} 1[\mathrm{~F}(1,59)=1,013, \mathrm{p}=$ $0,318]$, nem grupo $G_{3}[F(1,59)=1,740, p=0,192]$.

Um resultado significativo foi visto no momento antes e depois do grupo $\mathrm{G}_{2}$ para o membro inferior esquerdo (MIE) $[F(1,59)=10,527, p=0,002]$, além 
de efeitos principais em $\mathrm{G}_{1}$ tanto para membro inferior direito (MID) $[F(1,59)=17,240, p=0,000]$, quanto para $\operatorname{MIE}[F(1,59)=28,831, p=0,000]$. Notou-se um efeito principal e um resultado estatisticamente significativos para o MIE, comparando-se os momentos antes e depois, para os grupos $\mathrm{G}_{1}[\mathrm{~F}(1,59)=$ $28,831, p=0,000]$ e $\mathrm{G}_{2}[\mathrm{~F}(1,59)=10,527, p=0,002]$, respectivamente. Houve um resultado significativo ao realizar uma comparação entre os membros direito e esquerdo no momento antes, para o grupo $\mathrm{G}_{2}[\mathrm{~F}(1,59)=7,335, \mathrm{p}=0,009]$, e no momento depois para o grupo $G_{3}[F(1,59)=5,324, p=0,025]$.

O gráfico 3 corresponde às médias e erro padrão da $F M$ de $G_{1}, G_{2}$ e $G_{3}$ nos membros direito e es- querdo, antes e depois do atendimento. No membro direito, a média encontrada antes do atendimento para $\mathrm{G}$ foi de $179,16 \mathrm{mmHg}( \pm \mathrm{DP} 39,28)$, $\mathrm{G} 2$ de $188,00 \mathrm{mmHg}( \pm \mathrm{DP} 50,46)$ e $\mathrm{G}_{3}$ de 176,80 $\mathrm{mmHg}( \pm \mathrm{DP} 38,40)$. A média encontrada depois para $\mathrm{G}_{1}$ foi de $166,66 \mathrm{mmHg}$ ( \pm DP 41,56), G2 de $186,70 \mathrm{mmHg}( \pm \mathrm{DP} 56,65)$ e $\mathrm{G}_{3}$ de $173,20 \mathrm{mmHg}$ $( \pm \mathrm{DP} 43,26)$. No membro esquerdo, a média encontrada antes para $\mathrm{G}_{1}$ foi de $178,26 \mathrm{mmHg}( \pm \mathrm{DP}$ $35,04), \mathrm{G}_{2}$ foi de $196,70 \mathrm{mmHg}\left( \pm \mathrm{DP}_{45}, 11\right)$ e $\mathrm{G}_{3}$ de $180,80 \mathrm{mmHg}( \pm \mathrm{DP} 38,83)$. Já média no momento posterior ao atendimento para $G_{1}$ foi de 163,21 $\mathrm{mmHg}( \pm \mathrm{DP} 39,94), \mathrm{G}_{2}$ foi de $185,70 \mathrm{mmHg}( \pm \mathrm{DP}$ $49,14)$ e $\mathrm{G}_{3}$ de $180,40 \mathrm{mmHg}( \pm \mathrm{DP} 31,97)$.

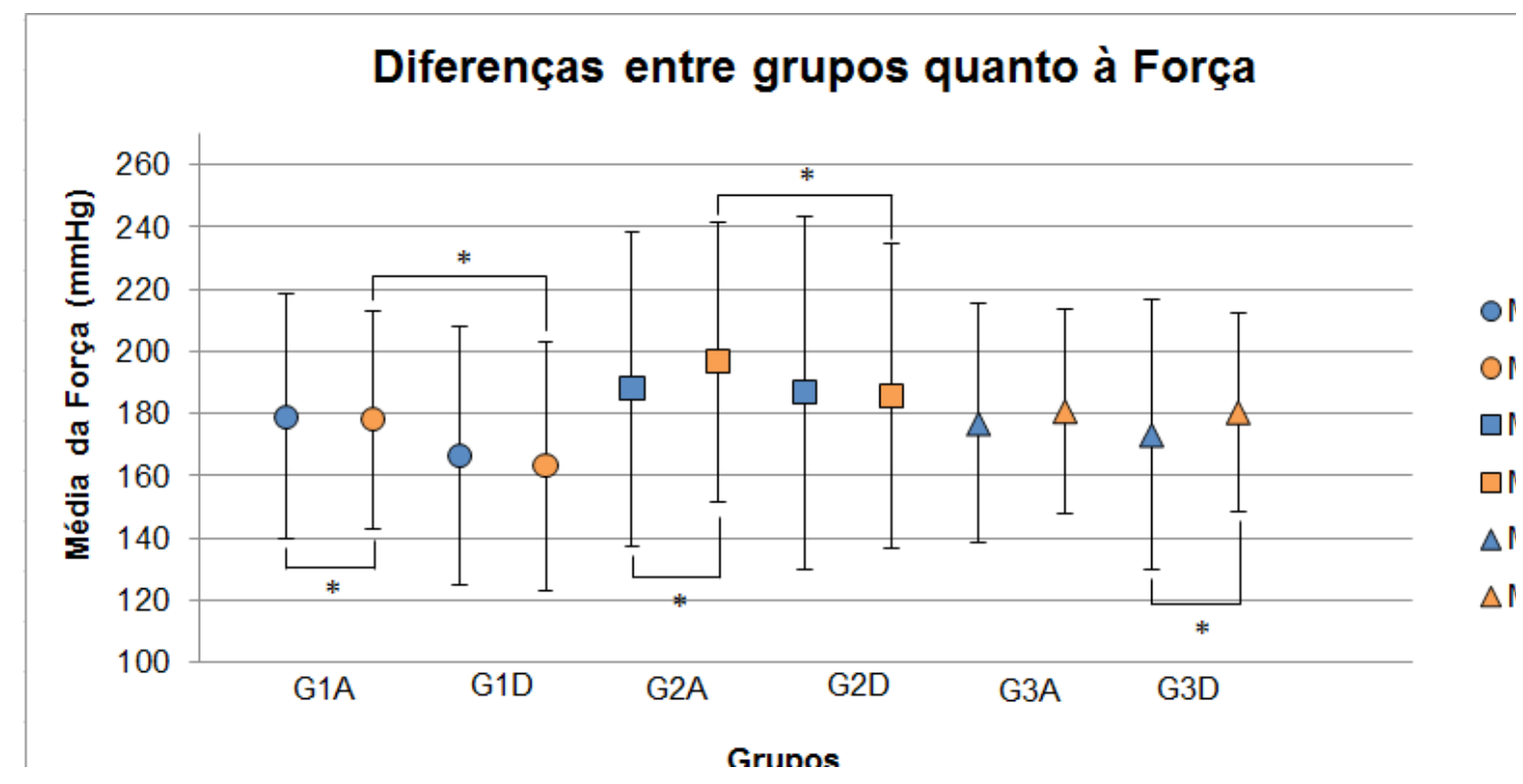

Gráfico 3 - Diferenças entre grupos quanto à Força

O gráfico 3 apresenta a média e erro padrão da FM avaliada no momento antes e depois da intervenção para o membro inferior direito (MID), em azul, e membro inferior esquerdo (MIE), em laranja. Os grupos $\mathrm{G}_{1} \mathrm{~A}, \mathrm{G}_{2} \mathrm{~A}$ e $\mathrm{G}_{3} \mathrm{~A}$ correspondem aos grupos de associação entre FNP e RV, somente FNP e somente RV no momento anterior; enquanto $G_{1} D, G_{2} D$ e $G_{3} D$, aos mesmos grupos no momento posterior ao atendimento. $\mathrm{O}$ asterisco (*) corresponde à diferença significativa com $p<0,05$.

\section{DISCUSSÃO}

\section{TEMPO DE REAÇÃO VISUAL}

Neste estudo, observou-se uma diminuição no tempo de realização do teste de reação pedal de Nelson para MMII nos três grupos avaliados após a intervenção, exceto para o MIE do $\mathrm{G}_{3}$, contudo, sem significância estatística. Para o TRV relacionado à RV, pode-se encontrar na literatura. Resul- 
tados semelhantes, ${ }^{(23)}$ como os do estudo realizado com dois grupos de participantes idosos, em que o primeiro participou, durante sete semanas, de jogos de videogame, como Donkey Kong e Pac Man, enquanto o segundo não jogou. Ao final, observou-se que os idosos que jogavam videogames eram mais rápidos do que os participantes do grupo controle. ${ }^{(23)}$ Esse achado tem relação direta com os achados do presente estudo. Green ${ }^{(24)} \mathrm{em}$ seu estudo, notaram que a experiência de jogos de ação em videogames aumenta o número de objetos que podem ser apreendidos, podendo monitorar maior número de objetos em movimento simultaneamente. Bem como em seu outro estudo, no qual participaram jogadores e não jogadores de videogame, que mostrou alteração na acuidade visual ou na capacidade de fazer discriminações finas de estímulos visualmente aglomerados, além da diminuição do tempo de resposta para jogadores em relação aos não jogadores. ${ }^{(25)}$ Outro estudo confirma os achados encontrados no presente estudo e os estudos citados acima; nele, os jogadores experientes foram capazes de rastrear objetos em movimento a velocidades maiores e executar com mais precisão um teste de memória visual a curto prazo, alternar entre as tarefas mais rapidamente e tomar decisões sobre objetos rotacionados com mais rapidez e precisão. ${ }^{(26)}$

$O$ atual estudo ressalta uma diferença média estatisticamente significante entre os grupos $G_{1}$ (FNP e RV) (colocar que tipo de grupo), G2 (FNP) e $\mathrm{G}_{3}$ (RV), mostrando melhores resultados no grupo que apresentou menor tempo de execução do teste. No entanto, ao comparar o grupo RV associado ao FNP, somente o FNP e apenas o RV, foi observado um decréscimo maior entre o grupo que houve apenas RV com o somente FNP, do que apenas RV com o grupo de associação RV e FNP. Isso mostra que o grupo $\mathrm{G}_{2}$ foi mais efetivo do que o $\mathrm{G}_{1}$, o qual foi superior aos resultados de $\mathrm{G}_{3}$. A RV também é utilizada na área da saúde visando recuperação e melhor desempenho funcional.

Um estudo comparativo entre indivíduos hígidos e portadores de Doença de Parkinson eviden- ciou que a RV não apresentou efeitos significativos na melhora do tempo de movimento e pico de velocidade. (27) Contudo, em outro estudo, publicado no mesmo ano, com uma amostra de 33 indivíduos similares ao estudo anterior, afirmou que a utilização da RV trouxe melhora significativa no tempo de movimento e aumento do pico de velocidade para o alcance de alvos móveis em membros superiores (MMSS). ${ }^{(28)}$ Durante o período em que esse estudo foi realizado não foram encontrados na literatura estudos que relacionassem a utilização da FNP com o TRV.

\section{EQUILÍBRIO DINÂMICO}

Um estudo feito com 16 idosos sedentários saudáveis, participantes de um programa de treinamento de equilíbrio com uso de exergames demonstrou melhora significativa no equilíbrio e marcha. ${ }^{(29)} \mathrm{Ou}$ tro estudo comparou o efeito de quatro semanas de intervenção utilizando um protocolo tradicional e outro com o exergame Wii Fit sobre o controle postural estático e dinâmico de participantes fisicamente ativos e com lesão prévia de MMII. Neste, foi observado maior desempenho do grupo Wii Fit no teste Time to Boundary para equilíbrio estático, sem, contudo, gerar diferença significativa entre os grupos na melhora do ED utilizando-se o SEBT. (30)

Um estudo realizado com 60 idosos demonstrou a eficácia da utilização de um programa de treinamento utilizando RV na melhora do equilíbrio e consequente redução do risco de quedas. ${ }^{(31)}$ O comprometimento da mobilidade e a redução do equilíbrio influenciam positivamente no aumento das chances de queda, por isso que treinamentos físicos tem se mostrado efetivos na melhora do equilíbrio e redução das quedas. ${ }^{(32,33)}$ Para a prevenção deste tipo de acidente, o treinamento muscular associado ao treino de equilíbrio tem demonstrado eficácia significativa. ${ }^{(34)} \mathrm{Em}$ um estudo, o Wii Fit promoveu aumento significativo do equilíbrio e FM em 10 mulheres saudáveis, com idades entre $30 \mathrm{e}$ 60 anos, ao serem submetidas a sessões de $30 \mathrm{mi}-$ nutos, 2 vezes por semana, durante 10 semanas. ${ }^{(35)}$ 
Analisando-se estudos ${ }^{(36,37)}$ mais específicos realizados em indivíduos não saudáveis, constata-se que a utilização de jogos de RV em indivíduos portadores de Doença de Parkinson traz melhorias significativas no equilíbrio e nas habilidades funcionais destes indivíduos. Esse achado tem relação direta com o estudo que ora se apresenta visto evidenciarem o mesmo resultado, o que reforça na literatura a aplicação da associação de FNP e RV. Quanto à associação entre FNP e ED, os resultados indicaram que os indivíduos submetidos à intervenção com FNP obtiveram melhoras significativas quanto ao desempenho de ED. A quantidade de atendimentos com intervenção de FNP pode ter influenciado nas melhorias que $\mathrm{G}_{2}$ alcançou corroborando com a literatura ${ }^{\left({ }^{8}\right)}$ que fala que a melhor resposta do paciente se dá ao longo dos atendimentos, e não somente em um único atendimento, para FNP.Acredita-se que a diferença encontrada no grupo FNP venha do fato do córtex cerebral armazenar padrões de movimentos (assim como no conceito FNP) e não movimentos isolados. Outro estudo efetuou intervenção com treinamento da estabilidade postural por meio de um protocolo fixo constituído por cinco exercícios utilizando o método FNP e observou diferença significativa ( $p<$ $\mathrm{O}, 01$ ), entre os valores pré e pós-teste, concluindo assim que a FNP obteve efeitos benéficos na estabilidade postural e em repercussões no risco de quedas em indivíduos com hemiparesia à esquerda. ${ }^{(39)}$ Em associação com o presente trabalho, este evidencia melhora funcional na estabilidade postural e redução dos riscos de queda, por maior estabilidade do participante após o treinamento.

\section{FORÇA MUSCULAR}

Neste estudo pode-se constatar que o $\mathrm{G}_{2}$ apresentou diminuição da FM em MIE e tal diferença não foi significativa no membro contralateral. Porém, antes dos atendimentos havia diferença entre ambos os membros, sendo o MIE significantemente mais forte que o MID, sugerindo que a intervenção proporcionou o nivelamento das forças por meio do enfraquecimento do membro mais forte. Tal achado contradiz um estudo realizado em 2009 que investiga os efeitos da FNP sobre a FM de MMII, mostrando que a técnica da FNP gerou, além do aumento da FM, ganho de flexibilidade e amplitude articular. (40) Cabe ressaltar que as técnicas de FNP utilizadas em ambos os estudos foram diferentes, o que pode ter influenciado na contradição. Outro estudo que avaliou a utilização da FNP sobre MMSS de mulheres idosas demonstrou ganhos significantes na FM. ${ }^{(41)}$

No $\mathrm{G}_{3}$ não houve diferença significativa na FM após as intervenções, porém houve alteração entre os membros, ficando o MIE mais forte que o contralateral, e havendo um desnivelamento entre as forças de ambos os membros, ou seja, o oposto do que foi observado no grupo que realizou intervenção apenas com FNP. Estudos que utilizaram jogos de RV de Nintendo Wii tanto em indivíduos hígidos quanto em indivíduos com sequelas de acidente vascular encefálico (AVE) constataram a melhora, não só da FM dos membros, como também da velocidade de movimentação e equilíbrio. ${ }^{35,42)}$ Outro estudo que também investigou os efeitos de jogos de RV sobre a FM de pacientes hemiparéticos crônicos pós AVE constatou que a intervenção aumentou significantemente diversas habilidades motoras, sendo uma delas a FM. ${ }^{(43)} \mathrm{Um}$ estudo que utilizou a RV com o intuito de melhorar a FM de flexores, extensores de cotovelo e abdutores de ombro em pacientes com lesão de plexo braquial obstétrico de Erb-Duchenne constatou que tal recurso não só trouxe benefícios neste quesito, como também na amplitude de movimento do membro acometido, dentre outras melhorias. ${ }^{(44)}$ Dos grupos avaliados neste estudo, nem os grupos com protocolos isolados, nem o grupo de associação RV e FNP foram efetivos para o aumento significativo da força dos MMII O presente estudo é realizado com indivíduos hígidos, quando faz uma relação com estudos que foram realizados com indivíduos com doenças específicas, tem que detalhar as possibilidades e não apenas citar os estudos. A relação pode ser feita principalmente quando existe uma escassez, mas tem que aprofundar a discussão. 
Ao expor o usuário a diversos estímulos sensoriais através dos exergames, levando em conta as diversas interações osteomioarticulares, os impactos são estendidos a mais de uma variável. Há evidências na literatura ${ }^{(45)}$ de diversos benefícios como melhora do equilíbrio estático e dinâmico e do controle postural, organização do espaço temporal, coordenação visuo-motora, ganho de força muscular, maior concentração, motivação, interação social e gasto calórico, os quais são mais evidentes ao se comparar os exergames aos videogames tradicionais. No atual estudo, foi observado que a utilização da RV e sua associação com o FNP diminuíram o TRV, não tiveram resultados signifativos para o equilíbrio e diminuíram a força de MMII. Um estudo ressalta que utilização da RV na fisioterapia pode andar em conjunto com o desenvolvimento tecnológico a evolução de novas perspectivas terapêuticas. Os jogos estimulam a competitividade do indivíduo mesmo em ambientes virtuais, fazendo com que o paciente se esforce e se dedique mais à terapia, além de apresentarem movimentos mais funcionais do que os movimentos isolados, raramente utilizados no cotidiano, o que também é benéfico para o paciente. Houve a possibilidade de efeitos adversos durante a execução das manobras, como tonteira, enjoo entre outros, porém nenhum dos sujeitos evidenciou alguma alteração aos pesquisadores. ${ }^{(46)}$

Os dados de força foram os últimos a serem coletados e o seu decréscimo pode ser indicativo de fadiga muscular, uma vez que as manifestações de fadiga têm sido associadas ao declínio da força muscular durante e após exercícios submáximos e máximos, à incapacidade de manter uma contração durante certo tempo, à diminuição da velocidade de contração e ao aumento do tempo de relaxamento musculares.

\section{CONCLUSÃO}

O presente estudo, a partir dos seus resultados, permitiu concluir que todos os grupos foram eficazes para a diminuição do tempo de reação visual, destacando-se o $\mathrm{G}_{2}$ por apresentar maior decréscimo e, consequentemente, maior agilidade. Quanto às outras duas variáveis, ressalta-se que nenhum dos três grupos mostrou-se eficaz para o ganho de equilíbrio dinâmico e de força em adultos jovens sedentários. A utilização da modalidade cume dos reflexos também pode ter sido fator determinante para o insucesso da proposta terapêutica, já que se observou na literatura a eficácia de outras modalidades utilizadas para ganho de equilíbrio e de força. Nesse sentido, observa-se que os objetivos do estudo foram alcançados, visto terem evidenciado que ocorre em cada grupo.

\section{LIMITAÇÕES}

A principal limitação do atual estudo foi o tamanho da amostra $(n=18)$, o permite considerar os resultados apenas para uma pequena população. Outras foram a adesão ao protocolo submetido, uma vez que apresentou tempo estendido de cinco semanas e o participante necessitaria de disponibilidade para as coletas, e o fato de realizar a coleta de três variáveis em um mesmo momento, o que pode gerar um resultado falso positivo. Assim, sugerem-se novas pesquisas a fim de reproduzir este experimento, utilizando maior número de indivíduos, de repetições semanais e/ou de tempo de acompanhamento, além de momentos específicos para coleta de cada variável, sem que haja possível interferência entre os resultados.

\section{REFERÊNCIAS}

1. Burdea GC. Virtual Rehabilitation: benefits and challenges. Methods Inf Med, 2003;42:519-23.

2. Adamovich SV, Fluet GG, Tunik E, Merians AS. Sensorimotor training in virtual reality: a review. Neuro Rehabilitation. 2009;25(1):29-44.

3. Bowman DA, Kruijff E, LaViola Jr JJ, Poupyrev I. 3D User Interfaces: theory and practice. Boston: Addison-Wesley; 2005. 
4. Sutherland IE. Sketchpad: a man-machine graphical communication system. Cambridge: MIT: University of Cambridge; 1963.

5. Ribeiro MWS, Zorzal ER. Realidade Virtual e Aumentada: aplicações e tendências. Minas Gerais: Sociedade Brasileira de Computação; 2011. p. 151.

6. Vieira GP, Araujo DFGH, Leite MAA, Orsini M, Correa CL. Realidade virtual na reabilitação física de pacientes com doença de Parkinson. Journal of Human Growth and Development. 2014;24(1):31-41.

7. Rizzo AS, Lange B, Suma EA, Virtual Reality and Interactive Digital Game Technology: New Tools to Address Obesity and Diabetes. J Diabetes Sci Technol. 2011;5(2).

8. Mortari DM, Mânica AP, Pimentel GL, Efeitos da crioterapia e facilitação neuromuscular proprioceptiva sobre a força muscular nas musculaturas flexora e extensora de joelho. Fisioter Pesqui. 2009;16(4):329-34.

9. Carvalho KR, Cabral RMC, Gomes DAGS, Tavares AB. O método Kabat no tratamento fisioterapêutico da doença de Alzheimer. Rev Kairós. 2008;11(2):181-95.

10. Souza RA, Cruz LG, Carvalho OS, Silva FF, Carvalho WRG. Respostas cardiovasculares agudas em ambiente virtualmente simulado pelo Nintendo Wii. Rev Bras Cineantropom Desempenho Hum. 2013;15(1):60-70.

11. Guimarães JM, Farinatti PT. Análise descritiva de variáveis teoricamente associadas ao risco de quedas em mulheres idosas. Rev Bras Med Esporte. 2005;11(5).

12. Almeida APPV, Veras RP, Doimo LA. Avaliação de equilíbrio estático e dinâmico de idosas praticantes de hidroginástica e ginástica. Rev Bras Cineantropom Desempenho Hum. 2010;12(1):55-61.

13. Alonso AC, Bronzatto Filho E, Brech GC, Moscoli F. Estudo comparativo do equilíbrio postural entre atletas de judô e indivíduos sedentários. Rev Bras Biomecânica. 2008;9(17).

14. Cardoso G. Biblioteca de Funções para Utilização do Kinect em Jogos Eletrônicos e Aplicações NUI. Vale Itajaí: Universidade UNIVALI; $2 \mathrm{O} 2$.
15. Lee GC, Effects of Training Using Video Games on the Muscle Strength, Muscle Tone, and Activities of Daily Living of Chronic Stroke Patients. J Phys Ther Sci. 2013;25(5).

16. Galna B, Jackson D, Schofield G, McNaney R, Webster M, Barry G, et al. Retraining function in people with Parkinson's disease using the Microsoft Kinect: game design and pilot testing. J Neuroeng Rehabil. 2014;11:60.

17. Delgado C, Fernando Filho J, Barbosa FP, Oliveira HB. Utilização do esfigmomanômetro na avaliação da força dos músculos extensores e flexores da articulação do joelho em militares. Rev Bras Med Esporte. 2004;10(5).

18. Varela PMF. Coordenação Motora em Indivíduos Portadores de Síndrome de Down Praticantes de Actividade Física. Porto: Universidade do Porto; 2006.

19. Marques RJR. Caracterização do sistema visual em jovens atletas. Braga: Universidade do Minho; $2 \mathrm{Ol2}$.

2O. Gribble PA, Hertel J, Plisky P. Using the Star Excursion Balance Test to Assess Dynamic Postural-Control Deficits and Outcomes in Lower Extremity Injury: A Literature and Systematic Review. J Athl Train. 2O12;47(3).

21. Karloh M, Santos RP, Kraeski MH, Matias TS. Avaliação do equilíbrio dinâmico em atletas de ginástica rítmica. Revista Digital, Buenos Aires, 2009;(14)136.

22. Filipa A, Byrnes R, Paterno MV, Myer GD, Hewett TE. Neuromuscular Training Improves Performance on the Star Excursion Balance Test in Young Female Athletes. J Orthop Sports Phys Ther. 2010;40(9).

23. Clark JE, Lanphear AK, Riddick CC. The effects of videogame playing on the response selection processing of elderly adults. J Gerontol. 1987;42:82-85.

24. Green CS, Bavelier D. Enumeration versus multiple object tracking: The case of action video game players. Cognition. 2006;101:217-245.

25. Green CS, Bavelier D. Action video game experience alters the spatial resolution of attention. Psychol Sci. 2007;18:88-94. 
26. Boot WR, Kramer AF, Simons DJ, Fabiani M, Gratton $G$. The effects of video game playing on attention, memory, and executive control. Acta Psychol 2008;129:387-398.

27. Wang CY, Hwang WJ, Fang JJ, Sheu CF, Leong IF, Ma HI. Comparison of Virtual Reality Versus Physical Reality on Movement Characteristics of Persons With Parkinson's Disease: Effects of Moving Targets. Arch Phys Med Rehabil. 2011;92(8):1238-45.

28. Ma HII, Hwang WJ, Fang JJ, Kuo JK, Wang $\mathrm{CY}$, Leong IF, et al. Effects of virtual reality training on functional reaching movements in people with Parkinson's disease: a randomized controlled pilot Trial. Clin Rehabil. 2011;25(10):892-902.

29. Sims J, Cosby N, Saliba EN, Hertel J, Saliba S, A. Exergaming and Static Postural Control in Individuals With a History of Lower Limb Injury. J Athl Train. 2013;48(3):314-325.

30. Wüest S, Borghese NA, Pirovano M, Mainetti R, Langenberg R, Bruin ED. Usability and Effects of naExergame-Based Balance Training Program. Games Health J. 2014;3(2):106-114.

31. Duque G, Boersma D, Loza-Diaz G, Hassan S, Suarez $H$, Geisinger D, et al. Effects of balance training using a virtual-reality system in older fallers. Clin Interv Aging. 2013;8:257-263.

32. Hagedorn DK, Holm E, Effects of traditional physical training and visual computer feedback training in frail elderly patients. A randomized intervention study. Eur J Phys Rehabil Med. 2010;46:159-68.

33. Howe TE, Rochester L, Neil F, Skelton DA, Ballinger C. Exercise for improving balance in older people. Cochrane Database Syst Rev. 2011;9(11).

34. Abate M, Di lorio A, Di Renzo D, Paganelli R, Saggini R, Abate G. Frailty in elderly: the physical dimension. Eura Medicophys 2007;43(3):407-15.

35. Schiavinato AM, Machado BC, Pires MA, Baldan C. Influência da realidade virtual no equilíbrio de paciente portador de disfunção cerebelar: estudo de caso. Rev Neurocienc. 2011;19(1):119-127.

36. Esculier JF, Vaudrin J, Bériault P, Gagnon K, Tremblay LE. Home-based Balance Training
Programme Using Wii Fit with Balance Board for Parkinson's Disease: A Pilot Study. J Rehabil Med. 2O12; 44(2):144-150.

37. Pompeu JE, Mendes FA, Silva KG, Lobo AM, Oliveira $T$ de $P$, Zomignani $A P$, et al. Effect of Nintendo $\mathrm{Wii}^{\mathrm{TM}}$-based motor and cognitive training on activities of daily living in patients with Parkinson's disease: A randomised clinical trial. Physiotherapy 2012;98(3):196-2O4.

38. Wang R. Effect of proprioceptive neuromuscular facilitation on the gait of patients with hemiplegia of long and short duration. Phys Ther. 1994;74(12):1008-15.

39. Lacerda NN, Gomes EB, Pinheiro HA, Efeitos da facilitação neuromuscular proprioceptiva na estabilidade postural e risco de quedas em pacientes com sequela de acidente vascular encefálico: estudo piloto. Fisioter Pesqui. 2013;2O(1):37-42.

4O. Mortari DM, Mânica AP, Pimentel GL. Efeitos da crioterapia e facilitação neuromuscular proprioceptiva sobre a força muscular nas musculaturas flexora e extensora de joelho. Fisioter Pesqui. 2009;16(4):329-34.

41. Souza LB, Paim CRP, Imamura M, Alfieri FM. Uso de um ambiente de realidade virtual para reabilitação de acidente vascular encefálico. Acta Fisiátrica. 2011;18(4):217-21.

42. Sardi MD, Schuster RC, Alvarenga LFC. Efeitos da realidade virtual em hemiparéticos crônicos pós-acidente vascular encefálico. Rev Bras Ciênc Saúde. 2O12;10(32).

43. Rodrigues BS, Pagnussat AS, Chiquetti SEM. Efeitos da Realidade Virtual em Paciente Adulto com Paralisia Braquial Obstétrica. Rev Neurocienc, 2O12;2O(4):567-575.

44. Campelo AM, Pellegrini AM. A realidade virtual na intervenção motora em crianças com transtorno do desenvolvimento da coordenação. Rio Claro: Universidade Estadual Paulista; 2013.

45. Hidrian A, Weyler I. Comparison of the effect of Cyriax cross friction masage and a Nintendo Wii - exercise program for the treatment of pain in chronic lateral epicondylitis. A Amsterdam: European School of Physiotherapy, Professional Assignment Project 2008;6.

46. Fitts $\mathrm{RH}$. The cross-bridge cycle and skeletal muscle fatigue. J Appl Physiol, 2008;104:551-58. 